\title{
Un dilemme de sexualité/sécurité : la logique identitaire américaine face à la menace épidémique en Afrique
}

\section{Anne-Marie D'Aoust}

\section{(2) OpenEdition \\ Journals}

Édition électronique

URL : http://journals.openedition.org/conflits/1543

DOI : $10.4000 /$ conflits. 1543

ISSN : $1777-5345$

Éditeur :

CCLS - Centre d'études sur les conflits lilberté et sécurité, L'Harmattan

Édition imprimée

Date de publication : 1 juin 2004

Pagination : 133-162

ISBN : 2-7475-7301-X

ISSN : 1157-996X

Référence électronique

Anne-Marie D’Aoust, « Un dilemme de sexualité/sécurité : la logique identitaire américaine face à la menace épidémique en Afrique », Cultures \& Conflits [En ligne], 54 | été 2004, mis en ligne le 08 janvier 2010, consulté le 30 mars 2021. URL : http://journals.openedition.org/conflits/1543 ; DOI : https:// doi.org/10.4000/conflits. 1543

Ce document a été généré automatiquement le 30 mars 2021

Creative Commons License 


\title{
Un dilemme de sexualité/sécurité : la logique identitaire américaine face à la menace épidémique en Afrique
}

\author{
Anne-Marie D'Aoust
}

1 En tant que discipline longtemps considérée comme «naturellement » objective, les Relations Internationales (RI) ont véritablement pris racine dans leurs propres discours en renvoyant à des notions clairement identifiées et souvent réifiées, comme la « sécurité nationale», les «Etats», la «souveraineté » ou encore l'« anarchie». On constate en effet que les branches de l'arbre généalogique de la discipline renvoient à autant de pères spirituels, et que ceux-ci ont puisé à même des textes fondateurs aussi déterminants que ceux de Thucydide pour nourrir leur corpus théorique. La notion de sécurité nationale, notamment, s'est vue naturalisée et ancrée pour de bon dans le discours politique. Si une conception fortement empiriciste et positiviste de la discipline ainsi que de la sécurité a sous-tendu toute la période de la Guerre Froide, la fin de cette dernière a toutefois marqué le début de sérieuses remises en question des fondations de la discipline. Face à l'inaptitude des théories dominantes (le néoréalisme et l'institutionnalisme néolibéral) à rendre compte des transformations culturelles et identitaires en cours, de nouvelles approches qualifiées de «radicales» ont émergé, notamment le féminisme et le postmodernisme. En déconstruisant les réalités dites «naturelles» de la discipline, ces approches ont soulevé des questionnements quant aux enjeux sécurisés et aux groupes identitaires brimés et marginalisés par les discours sécuritaires. Le féminisme et le postmodernisme ont ainsi établi un lien étroit entre politique, sécurité, genre et identité, et ont permis d'avancer que la sécurité n'est pas simplement associée à la protection de l'Etat : elle est partie intégrante d'un état d'être (state of being).

2 L'élargissement du concept de sécurité ${ }^{1}$ a permis d'aller au-delà de la simple sécurité étatique pour tendre vers une idée de sécurité humaine ${ }^{2}$. C'est dans cette logique de 
sécurité humaine que le sida est progressivement passé, dans les discours politiques, d'un enjeu de santé publique à un enjeu sécuritaire. Ce transfert n'est pas fortuit: "Introduire un enjeu dans l'ordre du jour sécuritaire d'un pays, c'est lui donner priorité " ${ }^{3}$ soulignait Ken Booth. Conséquemment, que l'Administration Clinton ait hissé l'enjeu du sida au niveau de la sécurité nationale représente un tournant, puisque c'était la première fois qu'un enjeu de santé était clairement identifié dans la Stratégie de Sécurité Nationale (SSN) comme une menace directe à la sécurité nationale des EtatsUnis. On remarque pourtant que la présidence Bush n'a pas suivi complètement cette tangente et a préféré aborder le problème sous une autre facette en affirmant que le sida représentait d'abord et avant tout un enjeu de santé publique ${ }^{4}$. Ce changement de discours a été accompagné de l'annonce de mesures gouvernementales importantes, notamment l'établissement du Emergency Plan for AIDS Relief. Ce plan comporte cependant plusieurs clauses conditionnelles que les milieux juridiques n'hésitent pas à qualifier de «morales " ${ }^{5}$, surtout à l'égard des femmes. Un tel plan n'existait toutefois pas sous l'Administration Clinton.

3 Pour les tenants des approches féministes et postmodernes, l'appropriation d'un enjeu par l'Etat ainsi que sa catégorisation comme enjeu de « sécurité nationale » permet une organisation et une circonscription de l'enjeu selon une dynamique visant à protéger et reproduire des structures (masculines) dominantes de pouvoir. Les politiques américaines vis-à-vis du sida représentent donc un défi intéressant pour les tenants des approches féministes et postmodernes. On déplorera toutefois un manque de ressources francophones sur le sujet, et ce, autant sur le plan empirique ${ }^{6}$ que théorique. A notre connaissance, aucune étude francophone n'aborde de front la question de genre et sida en s'attardant spécifiquement sur le cas américain. De plus, bien que la campagne française de 2001 ait sous-entendu que la lutte contre le sida au féminin restait étroitement liée au féminisme ${ }^{7}$, force est d'admettre que cette piste est restée peu explorée. Les études francophones qui approfondissent le sujet sont rares, alors qu'une vaste littérature anglophone est disponible. On retrouve néanmoins quelques ouvrages et études francophones qui s'attardent sur les images véhiculées dans les médias relativement aux femmes séropositives ${ }^{8}$, mais peu adoptent un cadre d'analyse proprement féministe.

Dans le cas qui nous préoccupe, il est légitime de se questionner, à savoir si la sécurisation du sida par l'Etat américain a été plus profitable aux femmes que son traitement en tant que simple enjeu de santé publique. Si l'instinct nous porte à répondre spontanément par l'affirmative, il n'en reste pas moins que la réalité apparaît beaucoup plus complexe. Certains a priori doivent être questionnés : qui l'Etat tient-il à protéger en sécurisant l'enjeu du sida? Qu'implique le concept de "santé publique »? A quel corps physique renvoie le corps politique? Aussi, afin de mieux comprendre comment le traitement discursif de l'enjeu du sida contient et reproduit des structures hiérarchiques de pouvoirs qui s'effectuent dans une logique masculiniste de protection de l'identité américaine, nous effectuerons une brève présentation du traitement politique et discursif de l'enjeu par l'Administration Bush actuelle.

5 Un rappel des prémisses de l'approche féministe postmoderne circonscrira notre cadre d'analyse. Cette brève incursion théorique nous permettra ensuite de comprendre comment les discours américains actuels sur le sida cherchent d'abord et avant tout à protéger une certaine identité de l'Etat américain en tant que structure politique masculine de pouvoir. L'accent sera particulièrement mis sur le plan d'aide développé 
sous l'Administration Bush, qui illustre bien le traitement du sida comme enjeu d'(in)sécurité aux Etats-Unis. Une analyse de la représentation du corps politique et de sa protection mettra finalement en lumière l'exploitation de la peur de l'Autre pour consolider l'identité américaine.

\section{La présentation américaine de la problématique du sida : structures de pouvoirs, constances et changements}

\section{Discours/politiques de l'Administration Clinton}

Dans la mesure où une bonne partie des politiques de la présidence Bush est liée aux actions de l'Administration Clinton précédente, nous croyons bon de donner un aperçu des principales politiques mises en place par les deux Administrations et d'exposer les décisions, discours et autres actions politiques qui nous semblent avoir été les plus déterminants quant à l'évolution du traitement de l'enjeu du sida. Il est bien évident qu'il s'avère impossible pour nous d'exposer en détails toutes les démarches entreprises par les Administrations Bush et Clinton en ce qui a trait de près ou de loin à la question du sida ${ }^{9}$. En ce sens, ce bref rappel servira simplement de base afin de nous permettre de concentrer notre analyse sur les politiques de la présidence Bush actuelle.

L'Administration Clinton fut le premier gouvernement américain à accorder une place importante au sida dans ses décisions politiques. Malgré le fait que plusieurs investissements, notamment dans le domaine de la recherche, aient été effectués au cours du premier mandat ${ }^{10}$, c'est au cours du second que les mesures les plus ambitieuses furent prises. En 1995, Clinton créa le Presidential Advisory Council on HIV and AIDS (PACHA). Outre la création de cette structure politique et bureaucratique, le président Clinton mit sur pied en 1999 le White House Office of National AIDS Policy. La création du premier conseil visait un objectif précis : fournir au président et à son administration une expertise académique externe au milieu politique capable de formuler des recommandations sur la manière dont le gouvernement fédéral devrait organiser ses réponses face à l'épidémie du sida ${ }^{11}$. Le deuxième conseil, pour sa part, se limitait surtout à l'étude et à la coordination des politiques concernant le sida à l'intérieur des frontières américaines. En 1998, le président Clinton proposa au Congrès une initiative de 156 millions de dollars visant à mettre sur pied des programmes de prévention et de traitement spécifiquement pour les minorités afro-américaines et hispaniques, fortement touchées par l'épidémie. En 1999, l'Administration Clinton a poussé un peu plus loin le traitement politique de la question du sida en annonçant officiellement qu'elle considérerait désormais la maladie comme une "menace à la sécurité nationale $»^{12}$. Cette affirmation donna l'impulsion nécessaire à l'entérinement de la résolution 1308 du Conseil de sécurité de l'ONU ${ }^{13}$ ainsi qu'à l'intégration du sida dans la stratégie de sécurité nationale de 1999. Dans ce dernier document, la menace restait cependant essentiellement associée au continent africain: "Des menaces sécuritaires transnationales sérieuses émanent de certaines régions d'Afrique, incluant du terrorisme soutenu par certains Etats (...) et des maladies infectieuses, plus particulièrement le sida. (...) L'Administration a fait de la bataille contre le sida et d'autres maladies une priorité pour l'action internationale et l'implication en Afrique $~^{14}$. Vu l'importance que représente la stratégie de sécurité nationale dans l'élaboration des enjeux sécuritaires et militaires américains, ce 
geste a évidemment revêtu une grande valeur politique symbolique. Ce bref aperçu, s'il ne permet pas d'identifier les contextes spécifiques dans lequel chacune des politiques a été implantée, permet néanmoins de dégager le fait que les deux mandats de l'Administration Clinton ont été caractérisés par une institutionnalisation politique de la question du sida, qui avait été peu présente jusque là. Progressivement, le sida est ainsi passé d'un enjeu de santé publique à un enjeu politique de sécurité nationale sous l'Administration Clinton. C'est cette structure qui a permis à George W. Bush de poursuivre en matière de législation.

\section{Discours/politiques de l'Administration Bush}

Si l'Administration Clinton s'est distinguée par une politisation de l'enjeu du sida dans une perspective sécuritaire, l'Administration Bush, pour sa part, a suivi une ligne de conduite plutôt divergente. Bien qu'elle ait maintenu les structures organisationnelles mises en place sous l'Administration Clinton, comme le PACHA, l'Administration Bush n'a amorcé aucune mesure majeure concernant l'enjeu du sida avant le discours sur l'Etat de l'Union de janvier $2003^{15}$. Au cours de ce discours, le président Bush a annoncé sa décision de lancer l'Emergency Plan for AIDS Relief (EPAR), qui consiste à fournir une assistance de 15 milliards de dollars étalés sur cinq ans dans des pays bien ciblés d'Afrique et des Caraïbes afin de lutter contre le virus ${ }^{16}$. Ce plan vise directement à prévenir sept millions de nouvelles infections (dont $60 \%$ surviendraient dans les pays ciblés), à traiter deux millions de personnes déjà infectées, ainsi qu'à fournir support et soins à plus de dix millions d'individus et orphelins du sida ${ }^{17}$. Ce plan n'a toutefois reçu l'approbation du Congrès qu'en mai 2003 et est aujourd'hui connu sous le nom de United States Leadership Against HIV/AIDS, Tuberculosis, and Malaria Act of 2003 (ci-après identifié comme le US Leadership Against HIV/AIDS Act). Ce document constitue véritablement la pierre d'assise de la politique de l'Administration Bush en matière de politique étrangère et de sécurité liée au sida.

Le US Leadership Against HIV/AIDS Act comporte de nombreuses clauses qui explicitent l'organisation des efforts américains contre le sida. Le document comporte plusieurs objectifs stratégiques, notamment favoriser l'abstinence sexuelle, encourager la monogamie, ainsi qu'éradiquer la prostitution, le trafic sexuel, le viol et l'exploitation sexuelle $^{18}$. La place des organisations religieuses est très importante dans ce document et celles-ci se voient accorder un rôle important dans l'organisation des politiques américaines: "Les organisations religieuses possèdent les infrastructures, l'expérience et le savoir-faire nécessaires pour mener à bien ces programmes. Elles doivent donc être partie intégrante des efforts américains ${ }^{19}$. De plus, il est stipulé qu'au moins le tiers des fonds devra être alloué à des campagnes de prévention axées sur l'abstinence avant le mariage $^{20}$. La méthode ABC (abréviation qui tient lieu pour Abtsain, Be Faithful et use Condom) est officiellement privilégiée comme méthode de prévention, en ordre prioritaire $^{21}$. Bien que le US Leadership Against HIV/AIDS Act souligne que le sida représente une menace à la sécurité, il est écrit clairement que le VIH/sida est d'abord et avant tout un enjeu de santé22. On notera toutefois qu'on ne retrouve aucune mention au sida dans la stratégie de sécurité nationale de 2002. En somme, on constate que sous l'Administration Bush, le sida est vu selon une perspective de santé publique avant d'être présenté comme un enjeu de sécurité, en plus de rester fortement institutionnalisé politiquement. 


\section{Le recours au discours " épidémique » pour domestiquer le corps de l'« Autre »}

10 Le discours épidémique justifie la sécurisation de l'enjeu sida au nom de la sécurité nationale. On notera toutefois que cette sécurisation ne s'est effectuée qu'en 1999, soit plus de quinze ans après la découverte du premier cas de sida aux Etats-Unis. Pourquoi avoir attendu si longtemps? Une analyse des politiques hégémoniques liées à l'usage d'une rhétorique de l'épidémie permet ici de comprendre comment a pu s'effectuer le glissement discursif du traitement de l'enjeu du sida de la sphère de la santé à la sphère sécuritaire. Au début des années 1980, le virus du sida s'est essentiellement développé au sein de la communauté gay. Dès 1983, des activistes gays ont tenté d'effectuer des pressions auprès des divers membres du Congrès afin que des fonds soient alloués pour les traitements et la recherche. Ces démarches sont largement restées sans écho. En fait, ce n'est que lorsque le virus a commencé à toucher d'autres segments «plus importants » de la population, plus particulièrement l'homme hétérosexuel blanc, que le discours sur l'épidémie a commencé à prendre forme ${ }^{23}$. La notion "d'épidémie » correspond à une hégémonie particulière, qui va notamment favoriser l'émergence de structures de pouvoir par le biais de discours faisant la promotion de «safe sex». Par "hégémonie", nous entendons ici une construction sociale qui semble légitime au point d'opérer comme point naturel d'orientation du discours social ${ }^{24}$. L'usage d'une rhétorique s'enracinant dans le concept d'épidémie a une vocation stratégique, dans la mesure où elle permet de domestiquer les pratiques sexuelles et d'identifier les comportements valorisés se rattachant au « Nous ». Une précision s'impose toutefois ici : vouloir mettre en lumière la vocation politique stratégique de l'utilisation du discours épidémique ne signifie pas pour autant qu'il faille nier l'étendue de la propagation du sida. Nous croyons en effet que c'est la relation entre l'humain et le monde qui ne peut être "objectivée "; le monde ne peut exister objectivement en dehors de l'utilisation (subjective) du langage. C'est pourquoi l'hégémonie du concept d'épidémie doit être déconstruite pour démontrer ce qui tente d'être domestiqué par ce discours. Comme le souligne Linda Singer: "Une épidémie émerge comme le résultat d'un discours social dominant à la lumière duquel des corps seront mobilisés, des ressources seront allouées et des tactiques de surveillance et de régulation seront justifiées ${ }^{25}$.

11 Le discours épidémique identifie ainsi un Autre qu'il importe de domestiquer à des fins de protection. Cet Autre, dans le discours sécuritaire américain, est souvent présenté comme étant les pays contaminés de l'Afrique subsaharienne. L'idée sous-jacente est que la maladie provient d'un manque d'autorité (Etats fragiles et anarchiques) et qu'il faut un contrôle pour y remédier (contrôle militaire et contrôle sur le corps). Ainsi, plusieurs discours associent directement la menace du sida à l'anarchie qui menace les Etats dits «stables», comme les Etats-Unis. George Tenet, ancien directeur de la CIA, l'énonce sans détours : «Le sida met toute une génération en déroute. Et ensuite vous avez des vagues de réfugiés. Et ensuite vous avez des désastres économiques. Et ensuite vous avez des guerres civiles qui requièrent l'exfiltration ainsi qu'une forme d'intervention, que vous le veuillez ou non ${ }^{26}$. Le sida représente une menace à la sécurité de l'Etat-nation en tant que structure; c'est dans l'intérêt de protéger celle-ci que le problème sida doit être adressé, si les Etats-Unis ne veulent pas se plonger dans un état anarchique comparable à celui de l'Afrique. Ainsi, devant le Sénat, le groupe de recherche du Center for Strategic and International Studies (CSIS) a présenté la situation en des termes très nets : "Les 
impacts du VIH/sida sur les infrastructures critiques qui assurent la sécurité, la stabilité et la viabilité de l'Etat-nation moderne sont multiples. Dans la grande majorité des pays en développement, surtout les pays africains, le $\mathrm{VIH} /$ sida mine l'ordre et les capacités militaires, la légitimité politique (...) ainsi que l'ensemble de la cohésion sociale ${ }^{27}$. Dans son discours du $1^{\text {er }}$ décembre 2003, le secrétaire d'Etat Colin Powell a repris cette rhétorique en affirmant : "Le sida ravage des pays entiers et déstabilise des régions entières du monde ${ }^{28}$. En somme, le discours sécuritaire américain lié au sida repose sur une mise en évidence d'un rapport dichotomique stabilité/anarchie. C'est par l'usage d'une rhétorique épidémique que ce rapport est cristallisé et cette rhétorique justifie certaines mesures de contrôle pour protéger certains corps tout en en régulant d'autres.

\section{Les approches féministes}

\section{Présupposés théoriques}

12 Cette idée de contrôle du corps par le biais d'un discours épidémique laisse entrevoir toute la pertinence d'une analyse féministe postmoderne pour tenter de comprendre les mécanismes de pouvoir permettant le maintien d'une certaine identité de l'Etat américain. Néanmoins, afin de bien comprendre les spécificités de cette perspective théorique, une brève remise en contexte des approches féministes au sein de la discipline des Relations Internationales semble indiquée. Malgré le fait que l'on puisse identifier une tradition féministe des Relations Internationales dès les années 1960 avec le développement des approches féministes libérales, encore aujourd'hui, le croisement des deux approches reste limité. Ce ne sont pourtant pas les développements théoriques qui manquent. En effet, Jacqui True souligne combien l'appellation « féminisme » regroupe des approches aussi variées que contradictoires : on peut ainsi parler de féminisme libéral, de féminisme postmoderne, de féminisme marxiste, d'écoféminisme, de féminisme culturel, de féminisme postpositiviste, etc. ${ }^{29}$. Ces approches ont toutefois toutes en commun la volonté de dénoncer l'évacuation de la question du genre lorsqu'il est question de Relations Internationales. En usant chacune d'une méthodologie qui leur est propre, ces approches cherchent à faire entendre la voix des femmes au sein de la discipline des Relations Internationales ainsi qu'à démontrer comment la sécurité des femmes peut être compromise autant par la violence physique que structurelle qui s'effectue à tous les niveaux politiques et sociaux. Le genre est privilégié sur le plan ontologique, ceci afin de comprendre comment les hiérarchies fondées sur cette catégorie ont un impact négatif sur les individus comme sur certains groupes $^{30}$. Malgré la diversité des approches, le genre est défini par les féministes de manière générale et symbolique comme étant un ensemble de caractéristiques variables socialement et culturellement construites (par exemple le pouvoir, l'autonomie, la rationalité et le public) que l'on associe de manière stéréotypée à une image de masculinitée ${ }^{31}$.

13 Le féminisme postmoderne correspond à une variante particulière du féminisme qui s'est essentiellement développée dans les années 1990. L'approche féministe postmoderne met l'accent sur la construction sociale et discursive du rapport à l'altérité et cherche à en analyser les effets de vérité tout en tentant de décentrer les postures masculines de domination ${ }^{32}$. Le féminisme postmoderne puise directement ses sources dans le postmodernisme tel que développé dans les écrits d'auteurs comme 
Michel Foucault, Jacques Derrida ou Jean-François Lyotard ${ }^{33}$. L'idée de mettre en lumière des structures de pouvoir qui marginalisent les voix féminines est présente, tout comme l'idée qu'il n'y a pas de fondements à la connaissance. L'épistémologie féministe postmoderne refusera l'idée d'un savoir objectif et tentera plutôt de répondre aux questions suivantes : qu'implique la connaissance? Qui peut savoir ? Qui a accès à la connaissance et où? Quelles implications ces diverses localisations ont-elles sur le savoir $^{34}$ ? Vouloir élaborer une théorie féministe postmoderne pose évidemment problème, puisque l'idée de théorie renvoie à plusieurs notions discutables d'un point de vue féministe : qu'est-ce qui constitue une théorie? Quelles sont les implications de l'usage de la théorie pour une analyse féministe, si l'on considère que certains énoncés regroupés sous l'appellation de "théorie » ont des racines clairement masculinistes et eurocentristes? La théorie peut-elle être distincte des formes actuelles des politiques, n'est-elle pas en soi une forme de politique, donc de contrôle ${ }^{35}$ ? On recense donc de multiples approches féministes postmodernes qui chercheront d'abord et avant tout à mettre en lumière les pratiques d'exclusion et les rapports dichotomiques mis en place au sein de la discipline.

14 C'est toutefois sur le plan épistémologique que le féminisme postmoderne se distingue des autres formes de féminisme. Contrairement aux autres approches, le féminisme postmoderne offre des visions alternatives à la réalité présentée. Il ne s'agit plus ici de se limiter à une étude sur les femmes à l'intérieur de domaines pré-délimités par les structures préexistantes, il s'agit véritablement de développer des recherches féministes sur l'organisation de la connaissance ${ }^{36}$. Comme le note J. Ann Tickner, " inclure le genre comme catégorie centrale d'analyse transforme le savoir d'une manière qui va au-delà de l'addition des femmes à l'analyse; surtout, quoique cela soit souvent mal compris, cela signifie que les femmes ne peuvent être étudiées à l'écart des hommes ${ }^{37}$. Ce qui distingue toutefois la position féministe postmodernes des autres variantes féministes est l'insistance à questionner l'objectivité des concepts, notamment celui de «femme ». Comme l'ont souligné de nombreuses études, l'idée de «femme " n'est pas la même d'une région à l'autre, d'un pays à l'autre ainsi que d'une époque à l'autre. Ainsi, il existe plusieurs points de vues à partir desquels on peut remettre en question des stratégies qui cherchent à discipliner ${ }^{38}$. Le féminisme postmoderne renferme ainsi une contradiction en soi, puisqu'il doit à la fois mettre " la femme » au centre de l'analyse tout en cherchant à déconstruire les concepts réifiés et pris comme préexistants, $\mathrm{y}$ compris le concept de "femme». Cette difficulté amène ainsi une certaine tension entre le féminisme et le postmodernisme. En effet, certaines féministes comme Wendy Brown $^{39}$ et Seyla Benhabib ${ }^{40}$ craignent que le postmodernisme ne mène au relativisme et n'en vienne à étouffer le projet féministe dans l'œuf en lui niant son projet identitaire particulier.

\section{Conception féministe postmoderne de la « sécurité nationale »}

15 Les Relations Internationales, plus particulièrement les études stratégiques, ont longtemps évacué la question de genre dans l'étude des phénomènes sécuritaires. Pourtant, des auteurs comme Carol $\mathrm{Cohn}^{41}$ et Iris Marion Young ${ }^{42}$ ont démontré de manière fort convaincante comment la notion de genre était cruciale pour l'analyse des discours stratégiques sur la sécurité nationale. Le fait que ce soient des féministes qui aient tenté d'introduire la notion de genre dans le champ des études de sécurité n'a rien de surprenant. En effet, les études stratégiques et de sécurité sont dominées par 
des hommes autant sur le plan intellectuel que virtuel et, comme le fait remarquer Jane Flax dans une grande variété de cultures et de discours, les hommes sont perçus comme détachés du genre ou non déterminés par celui-ci. Les femmes se retrouvent donc avec la responsabilité de penser à la question du genre, mais parce qu'elles le font, leur travail se retrouve dévalué ou exclu de la vie intellectuelle conventionnelle et dominante ${ }^{43}$

Malgré tout, la création d'un sous-champ (marginal) d'études féministes en Relations Internationales ne témoigne pas tant d'une véritable intégration que d'une inclusion partielle ${ }^{44}$, puisque les ouvrages féministes sont très peu cités par les auteurs renommés du champ. Les études féministes sont notamment perçues par Stephen Walt comme "subversives» et menaçantes pour "l'intégrité et la cohésion intellectuelle» du champ ${ }^{45}$. En effet, les féministes postmodernes ébranlent sérieusement les fondations des études stratégiques traditionnelles en alléguant que l'Etat représente une source d'insécurité plutôt que de sécurité pour les femmes. Loin de représenter une structure sécuritaire, le système interétatique actuel est une source profonde d'insécurité systémique pour les femmes, autant sur le plan interne qu'externe ${ }^{46}$. Or, les théories traditionnelles de RI, particulièrement le néoréalisme, ont défini la sécurité en des termes politiques et militaires visant la protection des frontières physiques de l'Etat ainsi que de ses valeurs ${ }^{47}$. L'approche féministe postmoderne remet donc en question le contrat social et cherche, dans un monde de plus en plus interconnecté et en quête de sécurité globale, à outrepasser le schème de pensée classique qui limitait la conception de la sécurité à une logique de sécurité nationale. Les récits linéaires au sein desquels dominent des figures souveraines, comme "l'individu», "l'Etat " ou "la sécurité nationale " sont réfutés. Le féminisme postmoderne cherche donc à faire entendre les voix féminines marginalisées sous de faux couverts d'objectivité : « La voix de la raison n'est donc jamais innocente (...) Privilégier théoriquement un côté de la rationalité moderne, (...) c'est s'engager dans une pratique d'exclusion (et parfois de terreur) qui renvoie à l'expérience vécue de l'autre côté - par celui qui n'a pas de voix (rationnelle) » ${ }^{48}$. Cette idée sous-entend que le pouvoir n'a pas qu'un caractère répressif : il est également productif, dans la mesure où il produit une (fausse) normalité.

Puisque l'Etat ne correspond pas à une structure objective et qu'il s'est constitué historiquement selon une logique masculiniste, faire d'un enjeu une question de " sécurité nationale » revient à le structurer de manière à consolider et à perpétuer des relations de pouvoir fondées sur le genre ${ }^{49}$. Comme le mentionne V. Spike Peterson " des relations que nous concevions autrefois comme étant privées ou plus ou moins sociales sont en fait infuses de pouvoir, habituellement d'un pouvoir inégalitaire soutenu par les pouvoirs publics (...) Quand l'Etat intervient, il le fait habituellement selon une idéologie patriarcale qui, au mieux, 'protège' les femmes tout en reproduisant simultanément des acquis masculinistes qui assurent le 'besoin de protection' des femmes $\aleph^{50}$. En somme, le féminisme postmoderne cherchera à déconstruire les politiques de sécurité d'un l'Etat en posant (notamment) les questions suivantes : qui s'agit-il de sécuriser ? Ces politiques reproduisent-elles une logique masculiniste de protecteur/protégé qui justifie un contrôle et/ou une exclusion des femmes? Présente-on des rapports dichotomiques entre le public et le privé, entre ce qui est moral et amoral ? Ces questions permettront d'illustrer le caractère subjectif des politiques sécuritaires de l'Etat ainsi que les structures de pouvoir que ces politiques sous-tendent. 


\section{Analyse de cas dans une perspective féministe postmoderne : pourquoi sécuriser le sida au nom de la sécurité nationale?}

\section{L'Etat américain comme figure masculine de contrôle : protection, prostitution et contrôle}

En nous rattachant à la même ligne de pensée que V. Spike Peterson, nous pouvons tenter de comprendre comment l'institutionnalisation politique de l'enjeu du sida aux Etats-Unis répond à une logique masculiniste. Il s'agit donc ici de voir comment l'idée de genre est centrale au traitement politique américain de l'enjeu du sida et comment elle s'exprime dans une logique de sécurisation d'une identité masculine de l'Etat. Tout d'abord, on remarque que les discours américains sur le sida répondent à une logique masculiniste de protection. On constate en effet qu'autant les politiques développées par Clinton que celles mises en place par Bush identifient les femmes africaines comme un groupe-cible à protéger. Le US Leadership Against HIV/AIDS Act le mentionne d'ailleurs spécifiquement: «Les Etats-Unis (...) doivent fournir une assistance aux pays de l'Afrique subsaharienne (...) afin de contrôler la crise par le biais de la prévention $\mathrm{du} \mathrm{VIH} / \mathrm{sida}$ (...), [et d'implanter ] des activités axées sur les femmes et les jeunes, y compris des stratégies pour protéger les femmes et prévenir la transmission du virus de la mère à l'enfant $»^{51}$. La logique masculiniste de protection renvoie à l'idéal de l'homme chevaleresque, qui se trouve en fait à être l'« Autre » du mâle dominateur qui menace la femme. L'image est celle d'un Etat protecteur envers les femmes, mais comme le souligne Iris Marion Young, "la relation subordonnée de ceux qui se trouvent protégés est centrale à la logique masculiniste de protection. En échange de la protection masculine, la femme concède une part critique de son autonomie et de sa capacité à prendre des décisions " $^{52}$.

La question de la prostitution témoigne de manière éloquente de cette logique de protection imposée aux femmes africaines. En effet, le US Leadership Against HIV/AIDS Act spécifie que les Etats-Unis doivent favoriser l'éradication de la prostitution et qu'aucuns fonds ne doivent servir à promouvoir ou à favoriser la légalisation ou la pratique de la prostitution ou du trafic sexuel, jugés dégradants pour la femme. Si, au premier regard, cette loi semble démontrer une sensibilité particulière à la condition féminine, une analyse plus attentive met toutefois en lumière son aspect paternaliste et moral. En effet, cette clause compromet les programmes de luttes contre le sida dans les pays où la prostitution (et non l'exploitation et le proxénétisme) est légale. En fait, le US Leadership Against HIV/AIDS Act interdit le financement des groupes qui favorisent une approche du travail sexuel axée sur la réduction des risques pour les travailleuses du sexe. Le US Leadership Against HIV/AIDS Act cherche plutôt à le rediriger vers des initiatives dites "de secours " visant à fermer les maisons de prostitution et à inciter les travailleuses du sexe à abandonner leur métier ${ }^{53}$. Or, cette mesure ne tient pas compte de la réalité de nombreuses femmes africaines souvent peu éduquées qui, pour plusieurs raisons, n'arrivent pas à gagner assez d'argent pour assurer leur survie. La notion de "prostitution » est plutôt complexe dans cette région du monde et plusieurs auteurs soulignent qu'il serait plus approprié de parler de "sexe de survie " plutôt que de prostitution dans plusieurs cas, puisque le commerce sexuel sert à gagner de l'argent pour rester en vie, pour nourrir ses enfants ou pour arriver à négocier une certaine sécurité physique et une protection ${ }^{54}$. 
20 L'attitude du gouvernement américain face à la prostitution est également très ambiguë. Si aucune étude poussée n'a été effectuée jusqu'ici sur les relations entretenues par les militaires américains et les prostituées africaines concernées par le US Leadership Against HIV/AIDS Act, les études menées par Cynthia Enloe sur les bases américaines aux Philippines démontrent à tout le moins que le gouvernement américain a pris plusieurs mesures pour réguler les rapports des soldats américains avec les prostituées locales au cours des années 1990. Ces mesures favorisaient des examens médicaux réguliers des prostituées ainsi que la tenue d'un registre de cellesci, comme le rapporte Enloe : "Les femmes ne disposant pas de licences fournies après une examen se voyaient interdire par les autorités locales de continuer à travailler (...). Les commandants des bases ont ensuite ordonné que les photographies des Philippines infectées soient épinglées sens dessus dessous sur le babillard des avis publics afin de servir d'avertissement aux soldats Américains $»^{55}$. Les militaires identifiés comme porteur du VIH ou d'autres MST n'étaient évidemment nullement obligés de le rapporter à la femme qui, d'après eux, les aurait infectés.

21 Le US Leadership Against HIV/AIDS Act condamne la prostitution et cherche à décourager les femmes de s'y adonner, mais nulle part ne retrouve-t-on des recommandations visant à décourager les soldats à recourir aux services des prostituées. En fait, on ne recense qu'une seule clause du US Leadership Against HIV/AIDS Act se rapportant uniquement aux hommes. Elle s'applique à la prévention et insiste sur le fait que des fonds devront être alloués pour favoriser une « aide dans le but d'encourager les hommes à être responsables dans leur comportement sexuel, dans l'éducation de leurs enfants ainsi qu'à respecter les femmes ${ }^{56}$. Pour cette seule clause touchant les hommes, on dénombre au moins une dizaine de clauses se rapportant aux femmes. Cette réalité vient ainsi confirmer l'idée de Catherine Waldby, pour qui «le corps de l'homme hétérosexuel est absent en tant qu'objet problématique dans les discours sur le sida, absent en tant que corps ayant besoin d'une éducation et d'une discipline spécifique $\aleph^{57}$. Il est intéressant de noter que cette seule clause fait appel à la rationalité et au contrôle sur soi que peut posséder l'homme sur son comportement sexuel. Les clauses relatives au comportement sexuel des femmes ne font jamais mention de cette idée de "responsabilité » et insistent surtout sur leur besoin de protection (venant notamment d'hommes « responsables ») : "Les femmes sont quatre fois plus vulnérables à l'infection que les hommes et les taux d'infection augmentent considérablement, notamment parce que plusieurs sociétés ne fournissent pas aux femmes pauvres et aux jeunes filles les protections sociales légales et culturelles contre les activités à risques qui les expose au $\mathrm{VIH} / \mathrm{sida}^{5} \aleph^{58}$. Au total, le US Leadership Against HIV/AIDS Act associe au moins cinq fois le mot «vulnerable » avec le mot «women ». En fait, alors que l'on cherche à contrôler les corps des prostituées africaines, on cherche à présenter le recours à leur service comme inévitable. Cynthia Enloe souligne ainsi que la présence de prostituées permet aux soldats de consolider leur identité de combattant et ceci expliquerait notamment l'intérêt de l'Etat à contrôler plutôt qu'à proscrire la sexualité des prostituées qui ont des contacts avec les soldats : «Les forces armées ont besoin de femmes pour maintenir les bases en place, mais elles ont besoin de ces femmes pour imaginer que chacun appartient à des catégories d'exclusion mutuelles $»^{59}$. 


\section{Corps politique/corps physique : la femme comme élément menaçant à contrôler}

L'idée que le sida représente une «menace à la sécurité nationale américaine » est un énoncé assez vague en soi. En fait, il s'agit de savoir : qui est sous-entendu dans l'idée de la «nation américaine » à protéger; sous quelles formes se présente la menace à la nation? Indéniablement, la «nation»/«population» renvoie à l'idée d'un corps politique général. L'usage populaire identifie l'expression «corps politique » à l'unité nationale, mais plus spécifiquement, le corps politique est une expression qui implique d'imaginer la nation (ou toute autre structure gouvernementale, une ville par exemple) selon des lignes anthropomorphiques ou organiques ${ }^{60}$. La notion de "santé publique " traduit d'ailleurs cette idée que la nation forme un seul corps physique unifié. Il n'en reste pas moins que la nation, en tant que corps politique, justement, ne peut être asexuée. Le corps politique est en fait un corps masculin implicite, malgré tout présenté comme un corps idéal neutre. La logique sécuritaire du contrat social démontre clairement que le corps politique sous-tend un corps masculin : "La conception de la sécurité comme survie de soi, comme rapport au self help dérive de théories de l'état de nature qui n'opposent que des hommes à des hommes - que l'on évoque Hobbes ou Rousseau. Si dans cet état de nature on réinstaure les femmes et les enfants, alors la conception de la sécurité n'est plus le self help. La survie d'un autre, son enfant par exemple, peut prendre une place primordiale. (...) Ceci serait anecdotique si cela ne fondait la prétention à un discours scientifique sur la sécurité (...) » ${ }^{61}$.

Le corps politique rend trouble la distinction entre les intérêts du corps masculin et ceux de l'intérêt national en général: «Les corps masculins peuvent donc prétendre à l'inclusion littérale d'autres intérêts corporels parmi les leurs, notamment des intérêts de santé, parce qu'ils représentent les corps qui semblent incarner l'ordre social souhaitable ${ }^{62}$. On comprend donc que l'appropriation par l'Etat de l'enjeu du sida est en soi une structure masculine de pouvoir qui vise à protéger le corps politique. Cette dynamique de protection s'opère par le biais d'un contrôle du corps féminin et de sa sexualité, présentés comme menace. On constate en effet que le corps de la femme est perçu comme un médium de transgression des frontières entre "l'anarchique " (soit la personne infectée) et le «stable » (la population non infectée). L'image de la prostituée africaine, évoquée plus haut, ou encore de la réfugiée qui se déplace d'un endroit à l'autre et propage la maladie, illustre l'idée de ce corps de transgression à contrôler. Ainsi, la femme est perçue comme un élément fluide et mobile qui cause le passage du virus d'un groupe infecté à un groupe non infecté. Parce que les femmes ont la possibilité d'entrer en contact avec des groupes dits "à haut risques", comme les hommes bisexuels, elle sont perçues comme le médium transitoire entre les groupes hétérosexuels et bisexuels permettant la propagation du virus ${ }^{63}$. Cette image est exacerbée par l'idée selon laquelle les femmes infectées représentent la principale voie de transmission du virus de la mère à l'enfant. C'est donc la femme qu'il s'agit de contrôler, puisqu'elle devient un corps mouvant vu comme menaçant pour le corps masculin prétendument stable.

24 Par le biais de politiques de santé publique visant à protéger le corps politique masculin, le gouvernement américain accorde une responsabilité féminine indirecte vis-à-vis de la protection du corps mâle hétérosexuel. La méthode de prévention $\mathrm{ABC}$, tout comme les campagnes de prévention Just Say No font porter le fardeau du 
comportement sexuel sécuritaire aux femmes. La virginité et la pureté de la femme se trouvent valorisées, ainsi que les structures patriarcales traditionnelles de pouvoir, comme la famille. Le US Leadership Against HIV/AIDS Act cherche à promouvoir « des modes de vie sains, incluant l'abstinence, le retardement des relations sexuelles, la monogamie [et] le mariage (...) " ${ }^{64}$. Les autres comportement sexuels ou mode de vie représentent donc des menaces au corps politique masculin. Or, comme l'ont souligné plusieurs féministes, la famille traditionnelle constitue souvent la plus grande structure d'insécurité pour les femmes, les cas de violence physique et verbale et de nonrémunération des tâches ménagères en faisant foi. L'idée de "modes de vie sains " renvoie directement à la santé publique, qui est l'expression du corps politique masculin. La mise en valeur de femmes mariées, monogames et vierges n'est pas innocente, puisque ces dernières peuvent entrer dans l'imaginaire corporel (masculin) de la «nation», de la "population générale » et qu'elles ne représentent pas un corps qui pourrait possiblement transmettre le virus d'un corps mâle à un autre. En ce sens, elles ne représentent pas une menace à la santé publique/au corps masculin. Toutefois les femmes ne correspondant pas à ces profils identitaires (les prostituées, les réfugiées, les femmes ayant de multiples partenaires, etc.) sont identifiées comme "population à risques", dans un rapport dichotomique population générale/ population à risques. Il devient donc évident ici que les modes de vie « sains » sont ceux qui maintiennent en place les structures masculines de domination en place, comme la monogamie et le mariage.

\section{L'abstinence sexuelle comme méthode normative de sécurisation du corps politique}

Pour prévenir la propagation de l'épidémie du sida, le US Leadership Against HIV/AIDS Act cherche à contrôler le corps féminin. Ce contrôle passe notamment par la méthode de prévention $\mathrm{ABC}$, présentée par l'Etat américain comme la seule valable et fiable pour endiguer le virus. Le gouvernement Bush mentionne spécifiquement que la méthode $A B C$ est efficace et doit être utilisée comme méthode préventive: "Le succès de l'Ouganda démontre que le changement des comportements reposant sur le modèle $A B C$ constitue une méthode efficace pour prévenir la propagation $d u V I H »^{65}$. Appelé à justifier cette limitation des méthodes de prévention au modèle $A B C$, le secrétaire d'Etat Colin Powell a conclu que "les programmes qui en valent la peine traitent de l'éducation, permettent d'éduquer les gens à l'abstinence, à la fidélité [et] à l'usage de contraceptifs. (...) Nous investirons seulement dans ces programmes qui permettront d'observer des résultats concrets $\|^{66}$. Ce "succès " est cependant loin d'être une évidence : selon une recherche menée par l'Université de Columbia et un sondage effectué par l'Université de Kentucky, les promesses d'abstinence avant le mariage (qui est la méthode de prévention prioritaire de l'Administration Bush) s'avèrent inefficaces, sont souvent brisées et augmentent les comportements à risques ${ }^{67}$. De plus, si le US Leadership Against HIV/AIDS Act fait mention du "succès ougandais ", il passe sous silence les politiques menées par la Thaïlande, qui n'avait pas instauré une politique de prévention $\mathrm{ABC}$, mais dont l'efficacité et les résultats fructueux ont été salués par ONUSIDA ${ }^{68}$. Le refus de considérer des politiques autres que celles permettant un contrôle du corps féminin est donc réel, puisque d'autres options efficaces existent. On remarquera ainsi que l'abstinence sexuelle des Africains est valorisée, et vue comme un «success story»: «En 1995, 95\% des Ougandais rapportaient n'avoir eu qu'un(e) seul(e) partenaire pendant l'année (...) 
Le succès de l'ouganda démontre que le changement des comportements, par le biais de l'application du modèle $A B C$, est un moyen efficace pour prévenir la propagation du VIH ${ }^{69}$. L'inclusion de cette statistique dans le US Leadership Against HIV/AIDS Act laisse perplexe : non seulement elle ne renvoie à aucune étude ou à un échantillonnage précis, mais elle fait sourire le lecteur qui doute qu'une proportion aussi élevée de la population se soit abstenue ou n'ait eu qu'un seul partenaire. Implicitement, cette valorisation de l'abstinence sous-tend un contrôle de la reproduction de la population africaine: il s'agit de contrôler le comportement de ces corps anarchiques, déstabilisants et potentiellement dangereux qui propagent le virus. Ce contrôle du corps vise particulièrement la femme africaine, puisqu'une clause spéciale de l'Act spécifie que le gouvernement américain ne versera aucun montant à tout organisme qui supporte ou participe à un programme de planification familiale ou d'avortement ${ }^{70}$.

\section{Protéger la nation/les forces armées}

L'idée de nation traduit l'idée d'un corps politique masculin à protéger, et ceci passe par l'institution des forces armées. On ne sera donc pas surpris de constater que les discours américains associent étroitement la menace du sida aux forces armées. Ainsi, même si le vice-président $\mathrm{Al}$ Gore a mis le sida à l'ordre du jour au Conseil de sécurité de l'ONU, la Résolution 1308 qui en est sortie vise explicitement à protéger les soldats en priorité. En fait, les seules recommandations concrètes qui vont au-delà du stade de l'observation font mention de "l'impact potentiellement dommageable du VIH/sida sur le personnel de maintien de la paix, incluant le personnel de soutien $»^{71}$ et $"$ encourage tous les Membres intéressés qui ne l'ont pas déjà fait à considérer le développement (...) de stratégies efficaces à long terme concernant l'éducation, la prévention, le dépistage volontaire et confidentiel, l'encadrement et le traitement de leur personnel comme une part importante de la préparation des troupes pour les opérations de maintien de la paix $\gg^{72}$.

L'idée de protéger les forces armées est intimement liée à l'idée de sécurité nationale américaine. Le fait que le président Clinton fasse du sida une question sécuritaire en 1999 donne donc une dimension non seulement publique, mais également militaire à la façon dont doit être traité l'enjeu du sida. Les études sur le sida acquièrent un caractère proprement «militaire » et le tournant sécuritaire est confirmé avec la création de deux groupes d'études entièrement consacrés à cette question au sein de deux des think tanks les plus influents auprès des instances gouvernementales américaines. Le HIV/ AIDS Task Force du CSIS est créé, ainsi qu'un groupe de recherche à la Brooking's Institution. L'expression « Task Force » traduit d'ailleurs bien l'emphase qui est mise sur la dimension militaire de l'enjeu. Parmi les mesures de protection de l'Etat américain/ corps politique masculin par le biais de l'armée, notons l'impossibilité d'avoir accès à des statistiques précises sur les taux d'infection au sein du personnel des forces armées américaines $^{73}$. Ce manque d'information laisse véhiculer la perception que le sida ne touche en aucune façon les troupes américaines et que les militaires constituent un groupe «pur » qui court le risque d'être contaminé par les femmes. On ne peut ainsi relever aucune donnée sur le nombre de femmes infectées par les soldats. Or, plusieurs études ont mis en lumière le fait que le taux moyen d'infection des soldats est plus élevé que le même groupe d'âge moyen au sein de la société civile, et ce, peu importe la région $\mathrm{du}$ monde. Cette constatation s'applique non seulement aux pays en développement, mais également aux Etats-Unis, à la Grande-Bretagne et à la France ${ }^{74}$. En Afrique, le taux d'infection des soldats est environ quatre fois plus élevé que la 
population civile et en période de guerre, le taux peut devenir jusqu'à cinquante fois plus élevé ${ }^{75}$. Plusieurs études de sécurité soulèvent d'ailleurs que le sida représente une menace en mettant en jeu la crédibilité des missions des paix. Ainsi, Richard Holbrooke, ambassadeur américain aux Nations Unies, semble renoncer à un contrôle de la sexualité des soldats en invoquant l'«état de nature »et s'inquiète plutôt de la crédibilité compromise des forces armées : «La nature humaine est la nature humaine (...) Peu importe où les troupes de maintien de la paix vont, elles attirent les prostituées.(...) Nous devons empêcher l'ironie suprême qui surviendrait si, en tentant de prévenir un conflit, les soldats de l'ONU propageaient une maladie encore plus mortelle que le conflit lui-même » ${ }^{76}$.

Finalement, on constate que la réalité du viol comme arme de guerre rend les femmes particulièrement vulnérables à la menace du sida. Or, le Us Leadership Against HIV/AIDS Act ne comporte aucune clause uniquement liée à la question de la lutte contre le viol. Le viol est fréquemment utilisé comme arme de guerre et est devenu une action de guerre organisée à des fins politiques ou stratégiques ${ }^{77}$. On estime par exemple qu'entre 200000 et 500000 femmes ont été violées au Rwanda en quelques semaines lors du génocide de $1994^{78}$. L'apparition du sida transforme ces pratiques barbares en pratiques génocidaires, puisque la possibilité que des soldats infectés violent des femmes dans le but avoué de les infecter afin d'infliger des torts à long terme à l'ennemi est bien présente $^{79}$. Le sida a cependant créé un nouveau lien entre le viol et le génocide qu'on ne saurait nier aujourd'hui. L'absence de mentions spécifiques à ce sujet rend l'Etat complice de ces pratiques, puisque l'idée que le viol constitue un phénomène "normal», quoique déplorable, de la guerre a été assimilée. Dans aucune analyse retrouve-t-on des informations, voire des indications sur la possibilité de viols commis par des soldats américains sur des femmes africaines. Rien ne laisse cependant croire qu'il s'agirait d'une pratique inexistante. Pour V. Spike Peterson, le silence à ce sujet rend l'Etat « indirectement » complice de ces pratiques par la promotion plus ou moins consciente d'idéologies masculinistes et hétérosexistes ${ }^{80}$. On constate donc que le sida est une menace particulière à la sécurité des femmes et que cette menace n'est pas seulement liée au développement de la maladie comme telle : elle entre véritablement dans un schème structurel.

\section{L'idée de genre et de nation dans les rapports dichotomiques discursifs}

L'image d'une Amérique bienveillante (masculine) devant secourir une Afrique vulnérable (féminine) est le pendant direct des rapports dichotomiques stabilité/ anarchie et masculin/féminin que l'on retrouve dans les discours américains sur le sida. On dénote en effet un certain colonialisme idéologique dans les discours du président Bush et les références à l'Amérique paternelle bienveillante devant aider l'Afrique vulnérable et anarchique sont nombreuses. Ainsi, dans le discours du 27 mai 2003 où il entérinait le US Leadership Against HIV/AIDS Act, les expressions renvoyant à l'Amérique bienveillante abondent : «(...) Notre nation se prépare à une grande mission de sauvetage ", "Nous avons une longue tradition de générosité au service de l'humanité ", "Nous avons une responsabilité morale à agir et nous agissons", "La souffrance de l'Afrique est grande. (...) Les Etats-Unis d'Amérique ont le pouvoir et la responsabilité morale d'aider ", "L'Amérique prend cet engagement pour une raison évidente, directement enracinée dans notre fondation. Nous croyons dans la valeur et la dignité de chaque vie humaine ${ }^{81}$. Pour leur part et par opposition, les mots associés à l'Afrique soulignent sa faiblesse et son besoin de 
protection : "combat désespéré" ", "continent en crise ", "cette grande nation lance un appel à l'aide ", "continent ravagé $~^{82}$ sont autant d'expressions utilisées pour désigner l'Afrique.

Ce rapport dichotomique rend évident le fait que le gouvernement américain insiste sur le caractère « extérieur » de la maladie. Pour illustrer ce rapport interne/externe, David Campbell reporte le cas du docteur Cory Servas, membre de la Presidential AIDS Commission, qui a déclaré qu'il était patriotique de passer un test de dépistage du sida et d'être déclaré négatif ${ }^{83}$. De plus, les discours marquants de l'Administration Bush en lien avec le sida (notamment ceux proclamés le $1^{\text {er }}$ décembre, Journée internationale du sida) ne font jamais mention de la situation du sida à l'intérieur du pays. Un rapport dichotomique interne/externe est conservé et il est frappant de constater combien, dans les documents officiels, on tient à consolider la dimension «externe » de la maladie. Ainsi, le premier rapport gouvernemental connu faisant état du sida en tant que menace à la sécurité, le Global Infectious Disease Threat and Its Implications for the United States émis par le National Intelligence Council en janvier 2000, ne manque pas d'indiquer que malgré la présence du virus au pays, la maladie n'en provient pas : «Le $\mathrm{VIH} /$ sida a d'abord été identifié aux Etats-Unis mais est originaire de l'Afrique subsaharienne $»^{84}$. Il est également intéressant de noter l'association qu'effectuent les militaires américains entre la maladie et la protection du territoire national (homeland) : « Répondre aux causes des problèmes sanitaires régionaux en Afrique doit devenir plus prioritaire pour notre sécurité nationale (...) L'éventualité de la propagation d'une maladie pandémique jusqu'à nos côtes est définitivement un enjeu stratégique de défense du territoire national $\aleph^{85}$. Cette affirmation semble présupposer que la maladie n'a pas atteint de proportions inquiétantes aux Etats-Unis, que le pays est "protégé ». Or, AIDS Action rapporte qu'environ 40000 nouveaux cas d'infection au sida sont enregistrés annuellement ${ }^{86}$ et que sur ces nouveaux cas rapportés, la majorité implique des Blancs : $42 \%$ des nouveaux cas, soit un peu plus que les Noirs non hispaniques représentant $38 \%$ des nouveaux $\mathrm{cas}^{87}$. AIDS Action rapporte également que $82 \%$ des nouveaux cas reportés étaient des hommes. Or, comme nous l'avons vu, le US Leadership Against HIV/AIDS Act vise une domestication d'abord et avant tout du corps féminin. Aucun discours présidentiel n'a identifié l'Américain Blanc comme pouvant représenter une menace à la sécurité de son propre territoire ${ }^{88}$.

31 Le traitement discursif par l'Administration Bush de l'enjeu du sida contient et reproduit des structures hiérarchiques de pouvoirs qui s'effectuent dans une logique masculiniste de protection de l'identité américaine. Une interprétation féministe postmoderne permet ainsi de mettre directement en relief combien l'Etat américain émerge comme une figure masculine de contrôle, ce qui échappe aux analyses traditionnelles. La logique masculiniste de protection est reprise dans le US Leadership Against HIV/AIDS Act et on constate à quel point le traitement de notions comme la prostitution et le viol répond inadéquatement à la réalité vécue par les femmes africaines, qui sont directement visées par l'Act. L'idée de " sécurité nationale » renvoie à la nation américaine, soit le corps politique, mais celui-ci n'est pas asexué. L'approche féministe postmoderne permet d'illustrer comment le corps politique est intimement lié au corps physique masculin qu'il importe de protéger contre des éléments menaçants, notamment la femme prostituée ou la réfugiée qui agissent comme médiums de transgression des frontières entre populations infectées et non infectées. Il ressort enfin de l'analyse que les politiques préconisées par l'Administration Bush 
protègent d'abord l'Etat et ses structures masculines de pouvoir, particulièrement les forces armées.

Une analyse discursive permet enfin de comprendre comment l'usage d'une rhétorique de l'épidémie permet à l'Etat de domestiquer le corps de l'Autre, ici entendu comme étant le corps "menaçant» de l'Africaine infectée. La mise en place de rapports dichotomiques dans les documents gouvernementaux et les discours officiels montrent une représentation de l'Amérique bienveillante devant secourir une Afrique faible et vulnérable. Ce rapport dichotomique conforte les Etats-Unis dans leur identité. Ainsi, le sida reste associé à une menace " extérieure " provenant d'Afrique, une région instable et anarchique. Les Etats-Unis, pour leur part, sont représentés comme un Etat aux structures fortes devant se protéger de la menace externe. Cette conception passe sous silence le fait que plusieurs milliers d'Américains souffrent actuellement du sida, notamment des Américains blancs. Les discours officiels ne présentent jamais l'Américain blanc comme une menace à la nation, et l' «homme hétérosexuel » n'est jamais identifié comme un groupe à risques ou devant nécessiter un certain contrôle par le biais de politiques publiques. Les politiques discriminatoires des Etats-Unis en matière d'immigration confirment d'ailleurs cette volonté d'associer la menace du sida à l'Autre, à l'Etranger. La rhétorique américaine implique que l'Autre menace le territoire national et il s'agit de s'en protéger.

33 L'approche féministe a infirmé de manière pour le moins spectaculaire la prétention des analystes du CSIS qui assuraient à des sénateurs américains que la «lutte contre le $V I H / s i d a(. .$.$) va au-delà de l'idéologie "{ }^{89}$. Si le féminisme postmoderne ébranle les conceptions traditionnelles de la sécurité en insistant sur les rapports dichotomiques liés à l'(in)sécurité, il faillit toutefois à intégrer d'autres notions dans l'analyse sur lesquelles il faudrait éventuellement se pencher. Par exemple, le refus des Etats-Unis de donner l'accès à des médicaments génériques aux pays pauvres africains suscite des questionnements qui dépassent la présente analyse. Une analyse féministe postcoloniale apporterait entre autres des éléments d'analyse intéressants qui mettraient en relief certaines structures économiques de pouvoir en jeu ici et que nous avons évacuées, notre analyse s'articulant essentiellement autour d'éléments discursifs.

L'analyse féministe ne doit toutefois pas amener à croire qu'il faille dénoncer systématiquement toute politique s'adressant aux femmes et favoriser l'inaction, sous prétexte que toute action serait discriminatoire. L'analyse féministe, loin de vouloir favoriser la procrastination dans un ordre arbitraire établi, cherche plutôt à remettre en question ce qui semble, au premier abord, entièrement bénéfique pour les femmes, afin d'améliorer les structures en place. Ainsi, notre étude s'est attardée sur les politiques mises en place par la présidence Bush concernant le sida. Cependant, nous considérons que le sujet, par son ampleur, mériterait d'être traité dans une perspective plus large, ne serait-ce que pour poser le problème aux conceptions traditionnelles de la sécurité ainsi qu'aux approches plus critiques. Les politiques de lutte contre le sida entreprises sous la présidence Clinton, dont nous avons très peu parlé, mériteraient également d'être analysées en parallèle avec celles de la présidence Bush, ce que nous n'avons pu effectuer ici. Il conviendrait d'expliquer, par exemple, pourquoi les politiques de Clinton qui ont officiellement fait du sida un enjeu de sécurité nationale semblent moins préjudiciables envers les femmes que les politiques de Bush, qui a pourtant refusé de faire du sida un enjeu officiel de sécurité nationale. Serait-il possible que sécuriser l'enjeu du sida ait favorisé une attitude moins répressive de l'Etat envers 
les femmes en rendant l'enjeu important? Si oui, cette réalité contredirait-elle la conception féministe postmoderne de la sécurité nationale en tant que structure répressive et marginalisante? Ces quelques pistes d'analyse ouvrent la voie à une démarche plus réflexive au sein même des approches féministes et représentent un défi pour les approches traditionnelles. Elles rappellent également combien politique et identité restent intrinsèquement liées et qu'analyser l'une implique le renvoi à l'autre. Comme le souligne avec beaucoup d'à-propos Judith Butler « la déconstruction de l'identité n'implique pas la déconstruction des politiques; elle établit plutôt comme politique les termes mêmes avec lesquels l'identité est articulée $»^{90}$. Face à une discipline enracinée dans ses conceptions fermées de la sécurité, une invitation à la réactivation de l'attitude critique, à la créativité et à l'ébranlement des préconceptions s'avère nécessaire. Plus qu'un verbiage abstrait destiné à confondre les lecteurs, les approches critiques comme les diverses formes de féminisme doivent être comprises comme une position de pragmatisme et d'acuité face aux structures de pouvoir en place. Cette conscience politique invite à l'action, à l'adaptation constante et à la résistance plutôt qu'à la résignation face à un présupposé état de fait du monde tel qu'il est.

\section{NOTES}

1. Pour une recension de l'évolution des études du concept de sécurité dans l'après-Guerre Froide, consulter Smith S., "The Increasing Insecurity of Security Studies: Conceptualizing Security in the Last Twenty Years ", in Croft S., T. Terriff (dir.), Critical Reflections on Security and Change, Londres, Frank Cass, 2000, pp. 72-101.

2. Le concept de sécurité humaine a été popularisé par Barry Buzan avec son livre People, State, and Fear: An Agenda for International Security Studies International Relations, publié en 1983.Pour une étude des diverses définitions de sécurité humaine ainsi que de son applicabilité politique, consulter Paris R., «Human Security : Paradigm Shift or Hot Air? », International Security, 27, automne 2001, pp. 87-102.

3. Booth K., "Security and Self: Reflections of a Fallen Realist ", in Krause K. et M.C. Williams (dir.), Critical Security Studies: Concepts and Cases, Minneapolis, University of Minnesota Press, 1997, p. 111.

4. " United States Leadership Against HIV/AIDS, Tuberculosis, and Malaria Act of 2003 ", $108^{\mathrm{e}}$ Congrès des Etats-Unis d'Amérique, Public Law 108-25 adoptée le 27 mai 2003, Congrès des EtatsUnis d'Amérique, 27 mai 2003, p.13. Voir le site: http://frwebgate.access.gpo.gov/cgi-bin/ getdoc.cgi?dbname=108_cong_public_laws\&docid=f:publ025.108

5. . «Etats-Unis - L'appui financier à la lutte mondiale contre le sida préconisera un agenda moral ", Revue canadienne VIH/Sida et droit, vol. 8, no. 2, août 2003, pp. 32-33. Voir le site : http:// www.aidslaw.ca/publications/publicationsdocFR.php?ref $=251$

6. Dans le cas de la France, on retrouve néanmoins quelques documents qui fournissent des données intéressantes pour une éventuelle comparaison entre les politiques américaines et françaises. On peut ainsi retrouver en ligne la Politique nationale de lutte contre le sida en France, 2001-2004, et des données épidémiologiques sur la surveillance de la progression de l'épidémie à l'adresse suivante http://www.sante.gouv.fr/htm/dossiers/sida02/2sida.htm.

Le rapport «La politique de lutte contre le sida, 1994-2000 : rapport de l'instance d'évaluation 
présidée par Christian Rollet », Conseil national de l'évaluation, Commissariat général du plan, Paris, Documentation française, 2003, est également disponible.

7. Voir ministère de l'Emploi et de la Solidarité, ministère délégué à la Santé et Comité français d'éducation pour la santé, Campagne de prévention du sida auprès des femmes, mars 2001. Voir le site : http://www.cfes.sante.fr/70000/dp/01/dp010300.pdf

8. Se référer notamment à Nengeh Mensah M., Ni vues ni connues? Femmes, VIH, médias, Montréal, Editions du Remue-Ménage, 2003, 221 p. et à Breton C., Médias et sida : l'information entre paradoxes et malentendu, Lyon, Edition Fondation Marcel Mérieux, 1998.

9. Néanmoins, pour le lecteur désireux d'obtenir un recensement historique de la surveillance du développement du sida par les instances américaines de santé publique, il est possible de consulter Stoto M.A, Public Health Surveillance: A Historical Review with a Focus on HIV/AIDS, Rand Corporation, octobre 2003. Voir le site: http://www.rand.org/pubs/drafts/DRU3074/ DRU3074.pdf

10. Mentionnons notamment la signature du National Institute of Health Revitalization Act de 1993, qui a permis d'augmenter de 67 \% les budgets alloués à la recherche sur le sida au bout de cinq ans. Pour un bref résumé des politiques entreprises par le gouvernement Clinton en ce qui a trait au sida, se référer au document du Office of National AIDS Policy, The Clinton/Gore Administration: "A Record of Progress on HIV and AIDS », juin 1999. Voir le site: http:// clinton2.nara.gov/ONAP/pub/hivacc.pdf.

11. Ibid., p. 6.

12. Plus exactement, c'est en janvier 2000 que le vice-président américain Al Gore a demandé au Conseil de sécurité de l'ONU de considérer la santé comme un enjeu de sécurité. C'était la première fois qu'un enjeu de santé était débattu en tant que problème sécuritaire au Conseil de sécurité. Krasno J., "Gore Names Health a Security Issue at the U.N. », Yale Daily News, 17 janvier 2000. Voir le site: http://www.yaledailynews.com/article.asp?AID=3200.

13. La Résolution stipule clairement que « (...) la pandémie de VIH/sida, si elle n'est pas enrayée, peut mettre en danger la stabilité et la sécurité ", Résolution 1398, adoptée par le Conseil de Sécurité lors de sa $4172^{\text {ème }}$ réunion, 17 juillet 2000, Nations Unies, Conseil de Sécurité, juillet 2000. Voir le site : http://ods-dds-ny.un.org/doc/UNDOC/GEN/N00/536/03/PDF/N0053603.pdf?OpenElement

14. Maison Blanche, «A National Security Strategy for a New Century ", décembre 1999. Voir le site : http://www.au.af.mil/au/awc/awcgate/nss/nssr-1098.pdf .

15. George W. Bush, « State of the Union Address », 28 janvier 2003.

Voir le site : http://www.whitehouse.gov/news/releases/2003/01/20030128-19.html

16. «Fact Sheet: The President's Emergency Plan for AIDS Relief », Maison Blanche, 28 janvier 2003.Voir le site: http://www.whitehouse.gov/news/releases/2003/01/20030129-1.html

17. Ibid.

18. « United States Leadership Against HIV/AIDS, Tuberculosis, and Malaria Act of 2003 », op. cit., Page 117 STAT. 718, section 101-Development of a Comprehensive, Five-Year, Global Strategy, point (a), sous-point (4), p. 4.

19. Ibid., Page 117 STAT. 714, point (19), p. 4.

20. Ibid., Page 117 STAT. 746, section 402 b), point (3), p. 37.

21. Ibid., Page 117 STAT. 715, section 2, point 20 C), p. 5.

22. Ibid., Page 117 STAT. 714, section 2, point (15), p. 4.

23. Singer L., « Bodies-Pleasures-Powers», in Butler J., MacGrogan M., Erotic Welfare: Sexual Theory and Politics in the Age of Epidemic, New York, Routledge, 1993, p. 118.

24. Ibid., p. 129.

25. Ibid., p. 117.

26. Schneider M. et M. Moodie, "The Destabilizing Impact of HIV/AIDS: First Wave Hits Eastern and Southern Africa ; Second Wave Threatens India, China, Russia, Ethiopia, Nigeria ", Center for 
Strategic and International Studies, CSIS HIV/AIDS Task Force, mai 2002. Voir le site : http:// www.csis.org/africa/0205_DestImp.pdf .

27. Ibid.

28. Powell C.L., « World AIDS Day 2003 », Maison Blanche, Washington DC, $1^{\mathrm{er}}$ décembre 2003, http://www.state.gov/secretary/rm/2003/26599.htm .

29. True J., « Feminism », in Burchill S., Linklater A. (dir.), Theories of International Relations, New York, St. Martin's Press, 1996, p. 212.

30. Tickner J.A, Gendering World Politics, Columbia, Columbia University Press, 2001, p. 48. Notons toutefois que certaines approches renvoient à d'autres ontologies, notamment le patriarcat.

31. Tickner J.A., «You Just Don't Understand : Troubled Engagements Between Feminists and IR Theorists ", International Studies Quarterly, 41, 4, 1997, p. 614.

32. Bigo D., "Genre et relations internationales », colloque AFSP «Genre et politique », 30 et 31 mai 2002, http://www.afsp.msh-paris.fr/archives/2002/genretxt/bigo.pdf.

33. Le lecteur comme l'auteur pourra trouver surprenant, quoique révélateur, qu'aucune figure féminine, à l'exception de Julia Kristeva, ne compte parmi les auteurs à la base de l'élaboration des courants postmodernes. Cette absence, même au sein d'une approche prônant la fin de la marginalisation, témoigne de manière éloquente de l'absence des femmes dans la majorité des disciplines de sciences humaines et sociales.

34. Sylvester C., "The Contribution of Feminist Theory to International Relations ", in Smith S., Booth K. et M. Zalewski, International Theory: Positivism and Beyond, Cambridge, Cambridge University Press, 1996, pp. 256-257.

35. Butler J. et J. Scott., citées dans Sylvester C., ibid., p. 256 .

36. Bouchard N., " Féminisme ", dans Macleod A., Dufault E., et F-G. Dufour (dir.), Relations internationales : théories et concepts, Montréal, Athéna, 2002, p. 66.

37. Tickner J.A., « You Just Don't Understand : Troubled Engagements Between Feminists and IR Theorists ", op. cit., p. 621.

38. Sylvester C., Feminist Theory and International Relations in a Postmodern Era, Cambridge, Cambridge University Press, 1994, p. 13.

39. Consulter notamment l'article de Brown W., «Feminist Hesitations, Postmodern Exposures », Differences, 3, 1, 1991, pp. 63-84.

40. Consulter Benhabib S., "Feminism and Postmodernism», in Benhabib S. et al., Feminist Contentions: A Philosophical Exchange, New York, Routledge, 1995, pp. 17-34.

41. Voir Cohn C., "Sex and Death in the Rational World of Defense Intellectuals ", Signs : Journal of Women in Culture and Society, 12, 4, décembre 1987, pp. 687-718.

42. Voir Young I.M., "The Logic of Masculinist Protection : Reflections on the Current Security State », Signs : Journal of Women in Culture and Society, 29, 1, 2003, pp. 1-26.

43. Voir Flax J., Thinking Fragments: Psychoanalysis, Feminism, and Postmodernism in the Contemporary West, Berkeley, University of California Press, 1990, pp. 23-24.

44. True J., op. cit., p. 212.

45. A cet effet, consulter Walt S.M., " The Renaissance of Security Studies », International Studies Quarterly, 35, 2, 1991, pp. 211-239.

46. Peterson V.S., "Security and Sovereign States: What Is at Stake in Taking Feminism Seriously? ", in Peterson V.S., Gendered States: Feminist (Re)Visions of International Relations Theory, Boulder et Londres, Lynne Rienner Publishers, 1992, p. 32.

47. Tickner J.A, « You Just Don't Understand: Troubled Engagements Between Feminists and IR Theorists ", op. cit., p. 624.

48. George J., Discourses of Global Politics: A Critical (Re)Introduction to International Relations, Boulder, Lynne Rienner Publishers, 1994, p. 161.

49. Peterson V.S., op. cit., p. 33.

50. Ibid., pp. 45-46. 
51. « United States Leadership Against HIV/AIDS, Tuberculosis, and Malaria Act of 2003 », op. cit., PAGE 117 STAT. 728, section 301, sec. 104A., point (b), p. 19.

52. Young I. M., op. cit., p. 4.

53. «Etats-Unis - L'appui financier à la lutte mondiale contre le sida préconisera un agenda moral », op. cit., p. 3.

54. Preston-Whyte E. et al., "Survival Sex and HIV/AIDS in an African City ", in Parker R.G. et al. (dir.), Framing the Sexual Subject: The Politics of Gender, Sexuality, and Power, Berkeley, University of California Press, 2000, p. 165.

55. Enloe C., The Morning After: Sexual Politics at the End of the Cold War, Berkeley, University of California Press, 1993, p. 149.

56. « United States Leadership Against HIV/AIDS, Tuberculosis, and Malaria Act of 2003 », op. cit., Page 117 STAT. 729, SEC. 104A, point (d)(1)(C), p. 20.

57. Waldby C., " AIDS, Women And the Body Politic ", in Alan R., Petersen A., Waddell C. (dir.), Health Matters : A Sociology of Illness, Prevention and Care, Buckingham, Open University Press, 1998, p. 336.

58. « United States Leadership Against HIV/AIDS, Tuberculosis, and Malaria Act of 2003 », op. cit., Page 117 STAT. 712 , SEC. 2- Findings, point (3) (B), p. 2.

59. Enloe C., Bananas, Beaches \& Bases:Making Feminist Sense of International Politics, Berkeley, University of California Press, 1989, p. 91.

60. Waldby C., op. cit., p. 339. Pour une discussion détaillée sur le féminisme en lien avec le corps politique, consulter Gatens M., "Corporal Representations in/and the Body Politics ", in Diprose R. et R. Ferrell (dir.), Cartographies: Postructuralism and the Mapping of Bodies and Space, Sidney, Allen \& Unwin, 1991.

61. .Bigo D., op. cit. pp. 6-7

62. Waldby C., op. cit., p. 342.

63. Chavigny C.A. et al., cité dans Waldby C., op. cit., p. 345.

64. « United States Leadership Against HIV/AIDS, Tuberculosis, and Malaria Act of 2003 », op. cit., Page 117 STAT. 716, point (22E), p. 6.

65. Ibid., Page 117 STAT. 715, point (20C), p. 5.

66. Cité dans Sexuality Information and Education Council of the United States (SIECUS), « Bush Visits Uganda and Praises 'ABC' Approach In Spite of Administration's Preference for Abstinence-OnlyUntil-Marriage », juillet 2003.

Voir le site: http://www.siecus.org/policy/PUpdates/arch03/arch030065.html

Le SIECUS fournit notamment une liste relativement complète des groupes de pression et organismes qui font de l'abstinence avant le mariage un enjeu central à leurs politiques. Il est possible de consulter cette recension à l'adresse suivante: http://63.73.227.69/pubs/fact/ fact0016.html

67. Ibid.

68. « HIV/AIDS as a Security Issue », ICG Report, Washington/Bruxelles, 19 juin 2001. Voir le site : http://www.usaid.gov/regions/afr/conflictweb/docs/aids_hiv.pdf

69. « United States Leadership Against HIV/AIDS, Tuberculosis, and Malaria Act of 2003 », op. cit., Page 117 STAT. 715, point (20D), p. 5.

70. « United States Leadership Against HIV/AIDS, Tuberculosis, and Malaria Act of 2003 », op. cit., Page 117 STAT. 737, point (4f), p. 29.

71. Résolution 1398, op. cit.

72. Ibid.

73. Certains pays, notamment la Tanzanie, invoquent des raisons stratégiques pour garder ces statistiques secrètes: il s'agit de ne pas informer l'ennemi de la vulnérabilité des troupes. Cependant, à notre connaissance, les Etats-Unis n'ont jamais invoqué une telle raison pour empêcher la divulgation de ces chiffres. Quelques vagues statistiques existent, mais elles sont peu 
détaillées. Ce vague pourrait s'expliquer par le fait qu'un defense bill autorise les forces armées américaines à congédier les militaires testés séropositifs. De plus, depuis 1985, les forces armées américaines exigent un test de dépistage pour les candidats au recrutement. Vraisemblablement, le manque de données répond à une logique de protection des forces armées et tend à développer l'idée que les forces armées américaines sont exemptes de cas d'infection.

74. Singer P.W., « AIDS and International Security », Survival, 44, 1, 2002, p. 147.

75. Ibid., p. 148.

76. Cité dans Bratt D., «Blue Condoms: The Use of International Peacekeeper in the Fight Against AIDS », Université de Calgary, automne 2002. Voir le site : http://www.informaworld.com/smpp/ content $\sim \mathrm{db}=\mathrm{all} \sim$ content $=\mathrm{a} 714002745$

77. Pour une brève discussion à ce sujet, consulter Paquin J., «L'utilisation du viol systématique comme arme de guerre : étude de cas entourant le conflit en ex-Yougoslavie », FéminÉtudes, 7, 1, 2002, pp. 21-24.

78. Singer P.W., op. cit., p. 153.

79. Ce lien entre sida et génocide reste toutefois à approfondir. Certaines organisations non gouvernementales comme Human Rights Watch ont ainsi recensé quelques témoignages de femmes affirmant que leur agresseur les violait dans le but avoué de les infecter du virus. Il est cependant intéressant et significatif de relever qu'au moins un témoignage d'un homme affirmant avoir été forcé de violer une femme séropositive a été recensé, ce qui ouvre la porte à des analyses plus poussées qui examineraient la symbolique et la dynamique complexe existant entre genre, sida et ethnicité, particulièrement dans des cas où le viol est utilisé comme arme de guerre.

80. Peterson V.S., op. cit., p. 46.

81. George W. Bush, « Remarks by the President on the Signing of H.R. 1298, the U.S. Leadership Against HIV/AIDS, Tuberculosis and Malaria Act of 2003 ", Dean Acheson Auditorium, U.S. Department of State, 27 mai 2003.

Voir le site : http://www.whitehouse.gov/news/releases/2003/05/20030527-7.html

82. Ibid.

83. Campbell D., Writing Security: United States Foreign Policy and the Politics of Identity, $2^{\mathrm{e}}$ Edition, Minneapolis, University of Minnesota Press, 1998, p. 180.

84. National Intelligence Council, The Global Infectious Disease and Its Implications for the United States, Etats-Unis, janvier 2000, http://www.odci.gov/nic/special_globalinfectious.html

85. William Fox Jr. C., "Phantom Warriors: Disease as a Threat to US National Security ", Parameters, vol. XXVII, automne 1997-1998, p. 127.

86. «AIDS Action: Policy Facts", janvier 2001. Voir le site: http://www.aidsaction.org/ legislation/pdf/ProfileoftheEpidemic.pdf

87. Ibid., p. 2. Il faut toutefois souligner qu'il est possible de regarder ces chiffres de manière relative: on dénote peut-être plus de blancs infectés que de noirs, mais si l'on considère le pourcentage de noirs infectés par rapport au bassin de population qu'ils représentent, on peut en arriver à la conclusion que le sida touche plus directement la communauté noire que la communauté blanche.

88. Comme le souligne David Campbell: "The boundary-producing effects of the discourse surrounding AIDS took a literal turn when the U.S. Immigration and Naturalization Service overruled the Health and Human Services Department and reinstated the presence of HIV as grounds for excluding tourists and immigrants from the United States. With over one million Americans already infected with the virus, such an exclusion conveys that the danger is outside the U.S, is a foreigner, a stranger », Campbell D., op. cit., pp. 87-88.

89. Morrisson J.S., "The CSIS Task Force on HIV/AIDS : Strengthening U.S. Leadership ", Mémo du directeur du CSIS Tak Force on HIV/AIDS aux sénateurs Bill Frist (R-Tenn.) et John Kerry (D- 
Mass.), 27 février 2003. Voir le site: http://www.csis.org/africa/HIVAIDS/ 030227_secondepp_statement.pdf

90. Butler J., Gender Trouble: Feminism and the Subversion of Identity, New York, Routledge, 1990, p. 148.

\section{RÉSUMÉS}

Initié avec l'Administration Clinton, le sida a peu à peu été abordé dans son traitement, à la fois comme une question de santé publique et comme un «nouveau » problème sécuritaire auquel les Etats-Unis doivent maintenant faire face. Cette transformation discursive contient plusieurs implications qu'il faut aborder. En adoptant l'optique du féminisme postmoderne, cet article cherche à analyser comment les représentations discursives américaines de la pandémie du sida en Afrique visent à protéger une certaine conception de l'identité masculine de l'Etat et des structures du pouvoir. Une attention particulière est prêtée aux liens qui s'établissent entre le corps politique et le corps physique dans les discours touchant au sida, et aux implications sur le plan du genre de la loi de 2003 intitulée «United States Leadership Against HIV/AIDS, Tuberculosis and Malaria Act » adoptée par l'Administration de George W. Bush.

Starting with the Clinton Administration, AIDS has progressively been treated both as a public health matter and as a "new» security issue that the United States now has to face. This discursive transformation contains several political implications that need to be addressed. Adopting postmodern feminism lenses, this article seeks to analyze how the American discursive representations of the African AIDS pandemic aim to protect a certain conception of the state's masculine identity and structures of power. Particular attention is paid to the links established between the political and the physical body in AIDS-related discourses and to the gendered implications of the " United States Leadership Against HIV/AIDS, Tuberculosis, and Malaria Act » of 2003 adopted under the George W. Bush Administration.

\section{INDEX}

Mots-clés : Sida, sécurité, relations internationales, corps politique, corps physique, genre

\section{AUTEUR}

\section{ANNE-MARIE D'AOUST}

Anne-Marie D'Aoust est candidate à la maîtrise en science politique à l'Université du Québec à Montréal. Ses intérêts de recherches comprennent les théories critiques de la politique globale et la formulation de la politique étrangère américaine. 